\title{
Hereditary Kidney Wilms Tumor
}

National Cancer Institute

\section{Source}

National Cancer Institute. Hereditary Kidney Wilms Tumor. NCI Thesaurus. Code C8496.

Familial embryonal neoplasm derived from nephrogenic blastemal cells. Several lines of

differentiation, including blastemal, stromal and epithelial, are usually expressed.

Comprises approximately $1 \%$ of Wilms tumors. (AFIP fascicle version 2.0 ) 